\title{
Crystal structure of KorA bound to operator DNA: insight into repressor cooperation in RP4 gene regulation
}

\author{
Bettina König ${ }^{1}$, Jürgen J. Müller ${ }^{1}$, Erich Lanka ${ }^{2}$ and Udo Heinemann ${ }^{1,3, *}$ \\ ${ }^{1}$ Max-Delbrück-Center for Molecular Medicine, Robert-Rössle-Straße 10, 13125 Berlin, ${ }^{2}$ Max-Planck-Institute \\ for Molecular Genetics, Innestraße 73, 14195 Berlin and ${ }^{3}$ Institute for Chemistry and Biochemistry, \\ Free University, Takustraße 6, 14195 Berlin, Germany
}

Received December 23, 2008; Accepted January 14, 2009

\begin{abstract}
KorA is a global repressor in RP4 which regulates cooperatively the expression of plasmid genes whose products are involved in replication, conjugative transfer and stable inheritance. The structure of KorA bound to an 18-bp DNA duplex that contains the symmetric operator sequence and incorporates 5-bromo-deoxyuridine nucleosides has been determined by multiple-wavelength anomalous diffraction phasing at $1.96-\AA$ resolution. KorA is present as a symmetric dimer and contacts DNA via a helix-turn-helix motif. Each half-site of the symmetric operator DNA binds one copy of the protein in the major groove. As confirmed by mutagenesis, recognition specificity is based on two KorA side chains forming hydrogen bonds to four bases within each operator half-site. KorA has a unique dimerization module shared by the RP4 proteins TrbA and KlcB. We propose that these proteins cooperate with the global RP4 repressor KorB in a similar manner via this dimerization module and thus regulate RP4 inheritance.
\end{abstract}

\section{INTRODUCTION}

Plasmids of the incompatibility group IncP are capable of transfer and stable inheritance in most Gram-negative bacteria (1). Besides various bacterial hosts, they can also transfer to yeast (2) and some higher eukaryotic cells (3). IncP-1 plasmids are divided into several subgroups, $\alpha, \beta, \gamma$ and $\delta(4,5)$. The best-studied family members share a common backbone and fall in the $\alpha$ and $\beta$ subgroups (6): RP4 (indistinguishable from RK2, R18, R68 and RP1) for IncP-1 $\alpha$ (7) and R751 for IncP-1 $\beta$ (8). Their regulatory circuit, which coordinates partition, replication and transfer functions, is controlled by the global repressor proteins KorA, KorB and TrbA, each repressing several operons, while each operon is regulated by more than one repressor (9).

The stable maintenance of low-copy genomes like RP4 (60 $099 \mathrm{bp}$ with four to seven copies per chromosome) is based on accurate DNA segregation before cell division. This active partition (par) system of RP4 is encoded in the complex kor $A B$ operon. It carries the genes of its transcriptional repressors KorA and KorB (7) as well as the gene responsible for incompatibility, inc $C$ (10). IncC is made in two forms by two different translational starts in the same reading frame (11). The inc Cl (full-length gene product of $364 \mathrm{aa}$ ) and inc C2 (encoding an $\mathrm{N}$-terminally truncated version of 259 aa) starting regions are overlapped by the start and stop codon of kor A, respectively, in the second reading frame. This remarkable korA/inc $C 1 /$ inc $C 2$ arrangement of overlapping genes, by tandem in-frame initiation regions or by using the coding capacity of a different reading frame is conserved throughout the P-plasmids (7). While KorA contains a HTH motif, IncC1 is neither a DNA-binding protein nor a transcriptional repressor.

KorA consists of 101 residues $(11306 \mathrm{Da}, \mathrm{pI}=10.4)$ and exists as a dimer in solution. It recognizes and binds a 12-bp symmetric operator $\mathrm{O}_{\mathrm{A}}$ (consensus sequence 5'-GTT TAG CTA AAC-3') found at seven promoter sites on RP4 with different affinities (12). At five promoter regions a KorB operator site $\left(\mathrm{O}_{B}\right)$ is also present, separated from $\mathrm{O}_{\mathrm{A}}$ center-to-center by $32 \mathrm{bp}$ (kor Ap), $33 \mathrm{bp}$ (trf Ap, klaAp), 36 bp ( $k f r A p)$ or 92 bp (kle $A p)$. Interaction of both proteins is important to accomplish strong repression. KorB acts very flexibly and cooperates with other repressor proteins from various distances with no particular helical position required $(13,14)$. KorA interacts with KorB directly through its C-terminus (15). This domain exhibits a high level of conservation between KorA and two other RP4 proteins, TrbA and KlcB $(16,17)$.

To elucidate the binding of the KorA repressor protein to its operator sequence and reveal the molecular determinants of gene regulation by KorA, we have analyzed the

\footnotetext{
*To whom correspondence should be addressed. Tel: +49 309406 3420; Fax: + 49309406 2548; Email: heinemann@mdc-berlin.de
} 
structure of the KorA homodimer complexed to an 18-bp oligonucleotide containing the operator binding site $\mathrm{O}_{\mathrm{A}}$. The architecture of the KorA-operator complex leads us to propose a model for gene regulation at $\mathrm{O}_{\mathrm{A}} / \mathrm{O}_{\mathrm{B}}$ promoters in which KorB binds to the KorA dimerization interface from various distances through interactions involving the KorB N-terminus, while both proteins bind their operator sites.

\section{MATERIALS AND METHODS}

\section{Recombinant expression, protein purification, complex formation and crystallization}

The coding region for the KorA protein (residues 1-101) was inserted into the bacterial expression vector pMS470 $\Delta 8$ (18). Mutant variants were generated using the QuikChange mutagenesis protocol (Stratagene, Heidelberg). Escherichia coli BL21 (DE3) carrying the appropriate wild-type (wt) or mutant kor $A$ expression plasmid was cultured at $37^{\circ} \mathrm{C}$. Transcription in cells was induced at $A_{600}=0.8$ by addition of $1 \mathrm{mM}$ isopropyl1 -thio- $\beta$-D-galactopyranoside. Growth was continued for $4 \mathrm{~h}$.

After harvesting, cells were resuspended in $20 \mathrm{mM}$ Tris- $\mathrm{HCl}$ (pH 7.5), $1 \mathrm{M} \mathrm{NaCl}, 10 \%$ (v/v) glycerol, $1 \mathrm{mM}$ DTT and $1 \mathrm{mM}$ Pefablock and lysed using a French Press. KorA and mutants were purified essentially according to (12). In the final purification step, the proteins were subjected to size-exclusion chromatography (Superdex 75, Pharmacia), equilibrated with $20 \mathrm{mM}$ Tris $/ \mathrm{HCl}(\mathrm{pH} 8.0)$, $100 \mathrm{mM} \mathrm{NaCl}$ and $3 \mathrm{mM} \mathrm{MgCl}_{2}$. The proteins migrate as a single peak and elute as dimers at a volume corresponding to $22 \mathrm{kDa}$. Proteins were further concentrated in a 5-K-cutoff Ultrafree Millipore membrane. The fulllength wt KorA was stable in solution as confirmed by mass spectrometry and analytical ultracentrifugation. It did not crystallize under any condition tested.

HPLC-purified 18-bp oligonucleotides $\left(\mathrm{O}_{\mathrm{A}}{ }^{*}\right.$, $5^{\prime}-\mathrm{C}^{\mathrm{Br}} \mathrm{U}^{\mathrm{Br}} \mathrm{U}$ GTT TAG CTA AAC A ${ }^{\mathrm{Br}} \mathrm{UT}^{-} 3^{\prime}$ and 5'-AAT GTT TAG CTA AAC AAG-3'), purchased from BioTeZ (Berlin), were used for crystallization. The brominated DNA was kept in the dark at all time. Strands were mixed in 1:1 ratio, annealed and purified on a gelfiltration column (Superdex 75, Pharmacia), equilibrated with $20 \mathrm{mM}$ Tris/HCl $(\mathrm{pH} 8.0), 100 \mathrm{mM} \mathrm{NaCl}$ and $3 \mathrm{mM} \mathrm{MgCl}_{2}$. KorA and DNA were mixed in a 2.5:1 molar ratio, incubated at $37^{\circ} \mathrm{C}$ for $20 \mathrm{~min}$, and subjected to size-exclusion chromatography to separate the KorA $-\mathrm{O}_{\mathrm{A}}$ complex at $33 \mathrm{kDa}$ from free DNA and protein. Ultracentrifugation confirmed that one KorA dimer binds to one $\mathrm{O}_{\mathrm{A}}$ site. The complex was concentrated to $3.8 \mathrm{mg} / \mathrm{ml}$.

Crystallization conditions were identified by the hanging-drop vapor diffusion method. The best crystals of the complex were obtained by mixing $2 \mu \mathrm{l}$ of KorA $-\mathrm{O}_{\mathrm{A}}{ }^{*}$ complex $(3.8 \mathrm{mg} / \mathrm{ml})$ with $1 \mu \mathrm{l}$ of reservoir solution of $0.5 \mathrm{M}\left(\mathrm{NH}_{4}\right)_{2} \mathrm{SO}_{4}, 0.1 \mathrm{M} \mathrm{Na}{ }^{+}$citrate $(\mathrm{pH}$ 5.6) and $1.0 \mathrm{M} \mathrm{Li}_{2} \mathrm{SO}_{4}$. Monoclinic crystals grew in 3-5 days at $20^{\circ} \mathrm{C}$ in the dark.

\section{In vitro binding assays}

The 436-bp DNA fragment containing $\mathrm{O}_{\mathrm{A}} 1$ was PCRamplified using RP4 as template. The primers used are $\mathrm{O}_{\mathrm{A}} 1$ cw $\left(5^{\prime}\right.$-AAT GTT AGT TAA CAG CTG TGT GGC TTC CCA TCG ACT AA-3') and $\mathrm{O}_{\mathrm{A}} 1_{-}$ccw (5'-AAT GTT AGT TAA AAG CTT ATC GGC GCG ATC CTG GCG-3'). The competitor DNA (348 bp, $745 \mathrm{bp}$ and $1082 \mathrm{bp}$ ) without the $\mathrm{O}_{\mathrm{A}}$ sequence was also generated by PCR.

The standard reaction mixture $(10 \mu \mathrm{l})$ contained $20 \mathrm{mM}$ Tris $/ \mathrm{HCl}(\mathrm{pH} 8.0), 100 \mathrm{mM} \mathrm{NaCl}$ and $3 \mathrm{mM} \mathrm{MgCl} 2 . \mathrm{We}$ used 0.15 pmol of each PCR construct throughout. The reactions were then incubated with purified protein at a molar ratio of $1: 50$ for $20 \mathrm{~min}$ at $37^{\circ} \mathrm{C}$. After incubation the reactions were analyzed by electrophoresis on a nondenaturing $7.5 \%$ polyacrylamide gel in $1 \times \mathrm{TBE}$ running buffer at $8 \mathrm{~V} / \mathrm{cm}$, stained with ethidium bromide.

\section{Isothermal titration calorimetry (ITC) measurements}

For ITC experiments, purified KorA and 18-bp oligonucleotides $\left(\mathrm{O}_{\mathrm{A}}, 5^{\prime}\right.$-CTT GTT TAG CTA AAC ATT-3' and 5'-AAT GTT TAG CTA AAC AAG-3'), were dialyzed against the buffer, $20 \mathrm{mM}$ Tris/ $\mathrm{HCl} \mathrm{pH} 8,100 \mathrm{mM} \mathrm{NaCl}$ and $3 \mathrm{mM} \mathrm{MgCl}_{2}$. Samples were degassed for $\sim 5 \mathrm{~min}$ by vacuum aspiration prior to loading, and all titrations were carried out at $310 \mathrm{~K}$. Experiments were performed on a VP MicroCal VP-ITC titration calorimeter (MicroCal Inc., Northampton, MA) using the VPViewer 2000 software for instrument control and data acquisition. KorA solution measuring $40 \mu \mathrm{M}$ was filled in a stirred (310 r.p.m.) reaction cell of $1.4 \mathrm{ml}$. Injections, each of $10-\mu 1$ volume (first injection always 5 - $\mu$ l volume) and 10 -s duration with a 4-min interval between injections, were carried out using a syringe filled with $200 \mu \mathrm{M}$ 18-mer $\mathrm{O}_{\mathrm{A}}$ (Figure 5C) solution. Thermogram analysis was performed using the software ORIGIN (version 7.0) provided by the manufacturer.

\section{X-ray data collection and processing}

Two crystals were frozen in liquid nitrogen, and the solution for cryoprotection contained the reservoir solution with $15 \%(\mathrm{v} / \mathrm{v})$ of glycerol. A two-wavelength MAD experiment was carried out at $100 \mathrm{~K}$ in the dark using synchrotron radiation at beamline BL 14.1 (19) at BESSY (Berlin) on a fast scanning $225 \mathrm{~mm}$ CCD-mosaic detector from MAR Research (Norderstedt). Following the fluorescence scan the program 'CHOOCH' (20) calculated the anomalous scattering curves, and the wavelengths for the peak and inflection point were chosen. Data processing and scaling were carried out with XDS (21). The refinement statistics are given in Table 1. The KorA $-\mathrm{O}_{\mathrm{A}}{ }^{*}$ complex crystallized in space group $\mathrm{P} 2{ }_{1}$ with cell parameters of $a=44.83 \AA, b=115.45 \AA, c=49.88 \AA$, $\beta=114.0^{\circ}$. The asymmetric unit contains one copy of the $33-\mathrm{kDa}$ complex, with a solvent content of $65 \%$.

\section{Structure analysis and refinement}

The structure of the complex containing KorA (1-101) and the 18-bp $\mathrm{O}_{\mathrm{A}}{ }^{*}$ oligomer, in which three thymine 
residues were replaced by 5-bromodeoxyuracil (Figure 4A), was solved by multiple-wavelength anomalous diffraction (MAD). The program HKL2MAP (22) located six heavy atom sites, differing in relative occupancy and anomalous contribution. The number and position of the bromine sites was the first indication of a 2-fold disorder of the $\mathrm{O}_{\mathrm{A}}{ }^{*}$ duplex.

To improve the quality of the electron density map, the density modification program DM (23) was used. The noncrystallographic symmetry (NCS) of the heavy atoms in the ASU permitted subsequent phase improvement by DM, including automatic solvent and NCS masking as well as solvent flattening. warpNtrace implemented in the ARP/wARP software package (24) automatically built a first protein model (chain A 4-31, A 34-54, B 25-37 and B 47-61). This model was then manually adjusted and extended in $\mathrm{O}(25)$. Superposition of the A and B chains of KorA in LSQKAB (26) helped extend the model (chain A 3-61 and B 12-62). It was refined using Refmac5 and loose NCS restraints (27). Five percent of the reflections were set aside for cross-validation, and $R_{\text {free }}$ was used to monitor model refinement strategy and progress. After five runs of warpNtrace an 18-mer DNA model (strands $\mathrm{E}$ and $\mathrm{F}$ ), created with MAKE-NA (http://structure.usc.edu/make-na/), was inserted manually into the positive $F_{\mathrm{o}}-F_{\mathrm{c}}$ difference density and adjusted, guided by three bromine positions. Water molecules were added to the model (chains A 3-93 and B 5-93) using ARP/wARP. Strong positive density was observed at the three terminal base pairs on both ends (Figure 4C). This was satisfied by superimposing the DNA duplex in two orientations, and fitting the second double strand into the density using the three nonoccupied bromine positions. The occupancy of all DNA atoms was adjusted to 0.5 , and the six bromine positions were set to 0.25 . Although the light-sensitive brominated oligonucleotides were kept in the dark during purification, crystallization and the MAD experiments, the heavy atom sites were thus not fully occupied. Final refinement steps resulted in $R_{\text {free }}$ and $R_{\text {work }}$ of $20.1 \%$ and $17.0 \%$, respectively (Table 1 ). Diffraction data and atomic coordinates are available from the RCSB Protein Data Bank under 2w7n.

The refined structure shows excellent stereochemistry according to PROCHECK (28) and WHATCHECK (29) and contains one KorA (chains A 2-95 and B 3-97) dimer, one complete 18-bp $\mathrm{O}_{\mathrm{A}}^{*}$ duplex with a 2-fold disorder, and 317 solvent molecules. Figures were generated with PyMOL (30). Surface charges were calculated with APBS (31) in a range from $-10 \mathrm{kT}$ (red) to $+10 \mathrm{kT}$ (blue).

\section{RESULTS}

\section{Structure determination}

We crystallized the full-length transcriptional repressor KorA (residues 1-101) bound to a pseudo-symmetric 18-bp oligonucleotide, $\mathrm{O}_{\mathrm{A}}{ }^{*}$, of sequence $5^{\prime}-\mathrm{C}^{\mathrm{Br}} \mathrm{U}^{\mathrm{Br}} \mathrm{U}$ GT TTAG CTA AAC A ${ }^{\mathrm{Br}} \mathrm{UT}-3^{\prime} \quad\left(\mathrm{O}_{\mathrm{A}}\right.$ consensus sequence underlined). Outside of the 12-bp operator sequence three 5-bromo-deoxyuridine nucleosides were introduced into one DNA strand, and the crystal structure was solved by MAD phasing at a resolution of $1.96 \AA$ (Figure 1).

Table 1. Data collection and refinement statistics

\begin{tabular}{|c|c|c|c|}
\hline \multicolumn{4}{|l|}{ Data collection } \\
\hline Wavelength (๕) & 0.91991 & 0.92004 & 0.91991 \\
\hline Total reflections $\mathrm{s}^{\mathrm{a}, \mathrm{b}}$ & $229922(19830)$ & $216313(18556)$ & $230648(20085)$ \\
\hline Unique reflections $\mathrm{s}^{\mathrm{a}}$ & $60942(7376)^{\mathrm{b}}$ & $60954(7347)^{\mathrm{b}}$ & $31085(3817)$ \\
\hline Completeness $(\%)^{\mathrm{a}}$ & $92.6(68.4)$ & $92.5(68.0)$ & $93.3(70.1)$ \\
\hline$<I / \sigma(I)>^{\mathrm{a}}$ & $32.2(12.7)$ & $37.1(11.4)$ & $42.5(17.4)$ \\
\hline \multicolumn{4}{|l|}{ Refinement } \\
\hline Resolution range $(\AA)$ & & & $50-1.85$ \\
\hline$R_{\text {work }}(\%)$ & & & 17.0 \\
\hline$R_{\text {free }}(\%)$ & & & 20.1 \\
\hline Reflections in $R_{\text {work }}$ & & & 32666 \\
\hline Reflections in $R_{\text {free }}$ & & & 1753 \\
\hline Protein and DNA & & & 2961 \\
\hline Water oxygens & & & 317 \\
\hline \multicolumn{4}{|l|}{ Ramachandran (\%) } \\
\hline Most favored & & & 98.8 \\
\hline Additional allowed & & & 1.2 \\
\hline
\end{tabular}

${ }^{a}$ Values in parentheses are for the highest resolution shell.

${ }^{\mathrm{b}}$ Friedel pairs not merged.

${ }^{\mathrm{c}}$ Multiplicity-corrected $R_{\mathrm{sym}}$ as defined by Diederichs and Karplus (41). 
The asymmetric unit of the crystal contains one KorA dimer bound to $\mathrm{O}_{\mathrm{A}}{ }^{*}$. The structure was refined to $R_{\text {work }}=17.0 \%$ and $R_{\text {free }}=20.1 \%$ (Table 1$)$. The electron density of the complex is well defined. Alternative side chain conformations were observed for Glu63 (chains A and B of KorA) as well as Glu25 and Asp64 (chain B). These three solvent-exposed residues are not involved in KorA dimerization or DNA binding, and therefore the arrangement of the complex is not affected. All protein residues could be built into the electron density apart from several $\mathrm{N}$ - and $\mathrm{C}$-terminal residues, so that the final model contains residues $2-95$ of chain $\mathrm{A}$ and 3-97 of chain $\mathrm{B}$.

\section{KorA structure}

The KorA monomer is composed of two distinct domains connected by a linker consisting of residues $66-69$. A fourhelical assembly $(\alpha 1$, residues $7-14 ; \alpha 2,21-31 ; \alpha 3,37-44$; $\alpha 4,48-65)$ forms the N-terminal DNA-binding unit. The predicted HTH motif is comprised of $\alpha 3$, the scaffold helix and $\alpha 4$, the recognition helix. On one side, the recognition helix is flanked by the three short helices $\alpha 1-\alpha 3$, going up and down perpendicular to $\alpha 4$. One right-twisted $\beta$-strand $(\beta 1,72-78)$ and the adjacent $\mathrm{C}$-terminal helix $(\alpha 5,80-97)$ form the dimerization module. The helix $\alpha 5$ is positioned antiparallel to $\beta 1$ resulting in a gripper-like shape (Figure 1A).
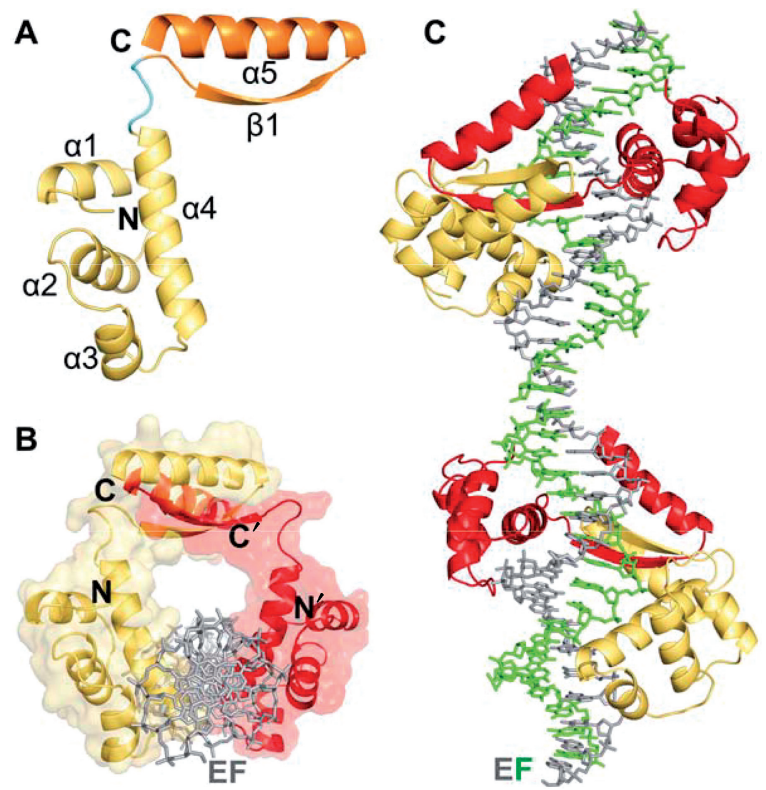

Figure 1. Overall structure of the KorA- $\mathrm{O}_{\mathrm{A}}{ }^{*}$ complex. (A) Schematic representation of the KorA monomer with the N-terminal DNAbinding domain (yellow), the linker (cyan) and the $\mathrm{C}$-terminal dimerization module (orange). (B) Image of the KorA dimer (yellow and red) on a semitransparent molecular surface bound to its operator site, looking down the DNA axis. For clarity, the DNA (gray) is shown in only one of the two orientations present in the complex. (C) Pseudocontinuously stacked operator DNA bound to the KorA dimer (yellow and red). The DNA is shown with strands E (gray) and F (green) of one duplex.

\section{KorA dimerization}

With their C-terminal gripper the KorA monomers interlock into a very tight homodimer. The $\beta$-strands associate to form an antiparallel $\beta$-ladder, and the C-terminal $\alpha$-helices form a two-helical bundle with an interaxial angle of $\sim 20^{\circ}$ (Figure 1B). In this arrangement, the helices stretch out along the curved surface of the $\beta$-sheet, and residues within the entire length of the $\alpha 5$-helices interact with residues of the $\beta$-sheet. Interactions modeling the dimer interface initiate in the linker region at residue Leu67 and continue toward the C-terminus of each monomer. The surface area buried in the dimerization interface covers $14.7 \%\left(2115 \AA^{2}\right)$ of the total solventaccessible surface area, ASA, $\left(14345 \AA^{2}\right)$ for both KorA monomers, calculated using the PISA server (32) with a probe radius of $1.4 \AA$. Across the interface, side chain interactions are hydrophobic in the core region with the tightly packed aliphatic side chains of Val74, Leu78 and Val86 (Figure 2A). The strongest van der Waals interactions $\left(\triangle \mathrm{ASA}>40 \AA^{2}\right)$ arise from residues Tyr71-Pro79 of the $\beta 1$-strand, and the $\alpha 5$ residues $G \ln 82$ and Val86-Glu90, symmetrically contributed by each monomer. The $\beta$-strands are connected to an antiparallel $\beta$-ladder by eight hydrogen bonds formed between opposite main chain atoms (Ala72, Val74, Ala76 and Leu78) and the hydrogen bonded side chains of Thr75 directly in the center of the sheet. Hydrogen bonding between backbone atoms of Gly70 and Glu80 positions the N-terminus

A
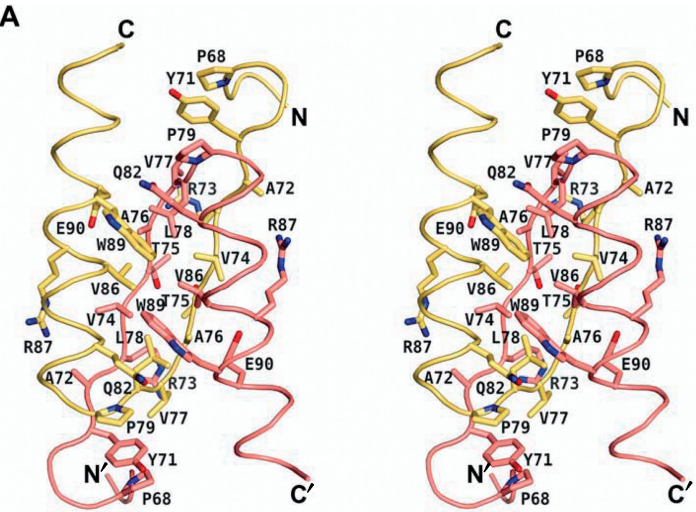

B

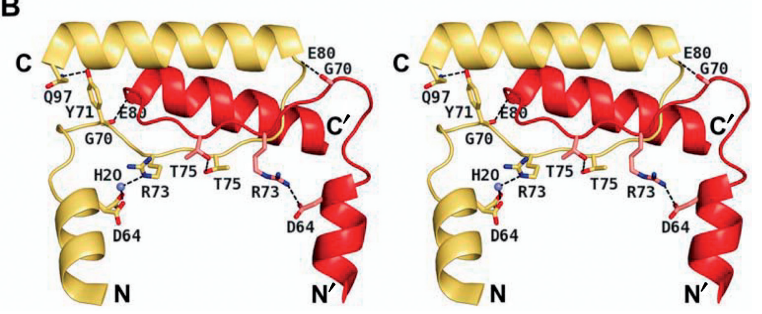

Figure 2. Interactions within the KorA dimerization module. (A) Stereo image of the KorA dimerization interface. All residues involved in dimerization are shown for both monomers (yellow and red). (B) Stereo representation of the KorA dimerization module (yellow and red). Residues involved in stabilization of the ring-like assembly are shown as sticks. 
of helices $\alpha 5$ and $\alpha 5^{\prime}$ on top of the N-terminus of the strands $\beta 1^{\prime}$ and $\beta 1$, respectively.

\section{Ring-like complex assembly}

In the crystal structure, the dimerization module of the KorA dimer is displaced from the DNA-binding domain, bound to its operator $\mathrm{O}_{\mathrm{A}}$. The C-terminal domain is elevated above the DNA-binding part of KorA and stabilized through various interactions. First, residues from the linker and dimerization region are involved in stacking-like van der Waals interactions. Side chains of Pro68-A, Tyr71-A and Pro79-B are arranged on top of each other in a sandwich formation with Tyr71-A positioned in the center (Figure 2A). This arrangement is continued with stacking-like van der Waals interactions of Gln82-B, contacting Pro79-B and Trp89-A. Due to the 2-fold pseudo-symmetry of dimeric KorA, these interactions occur twice in the interface. Second, the hydrogen bonded side chains of Asp64 and Arg73, each belonging to a different subdomain within one KorA monomer, are stabilizing the assembly. In chain B this interaction is water mediated (Figure 2B). The spatial separation of the dimerization subdomain from the major groove of the DNA results in a ring-like appearance of the complex assembly with an inner diameter of $\sim 20 \AA$ (Figure 1 B).

In solution, the ring-like assembly of the KorA-DNA complex may be expected to be deformable, and a flexible attachment of the C-terminal domain to the DNA-bound core of the protein is most likely biologically relevant. KorA is known to cooperate with another major RP4 repressor protein, KorB, via its C-terminal domain from various distances (33). For these direct interactions the ring-like assembly could potentially serve as an adaptable anchor site.

\section{Proposed KorA-KorB interaction site}

To understand the interaction between KorA and KorB in transcriptional regulation of RP4 genes we aligned the sequences of RP4 and R751 proteins KorA, TrbA and KlcB, all known to cooperate with KorB (16) using CLUSTALW (34). KorA and TrbA are global repressors in these plasmids, but the function of $\mathrm{KlcB}$ is still unknown (17). Over the length of the matched sequences, a variable degree of sequence conservation is observed (Figure 3A), indicating similar

A
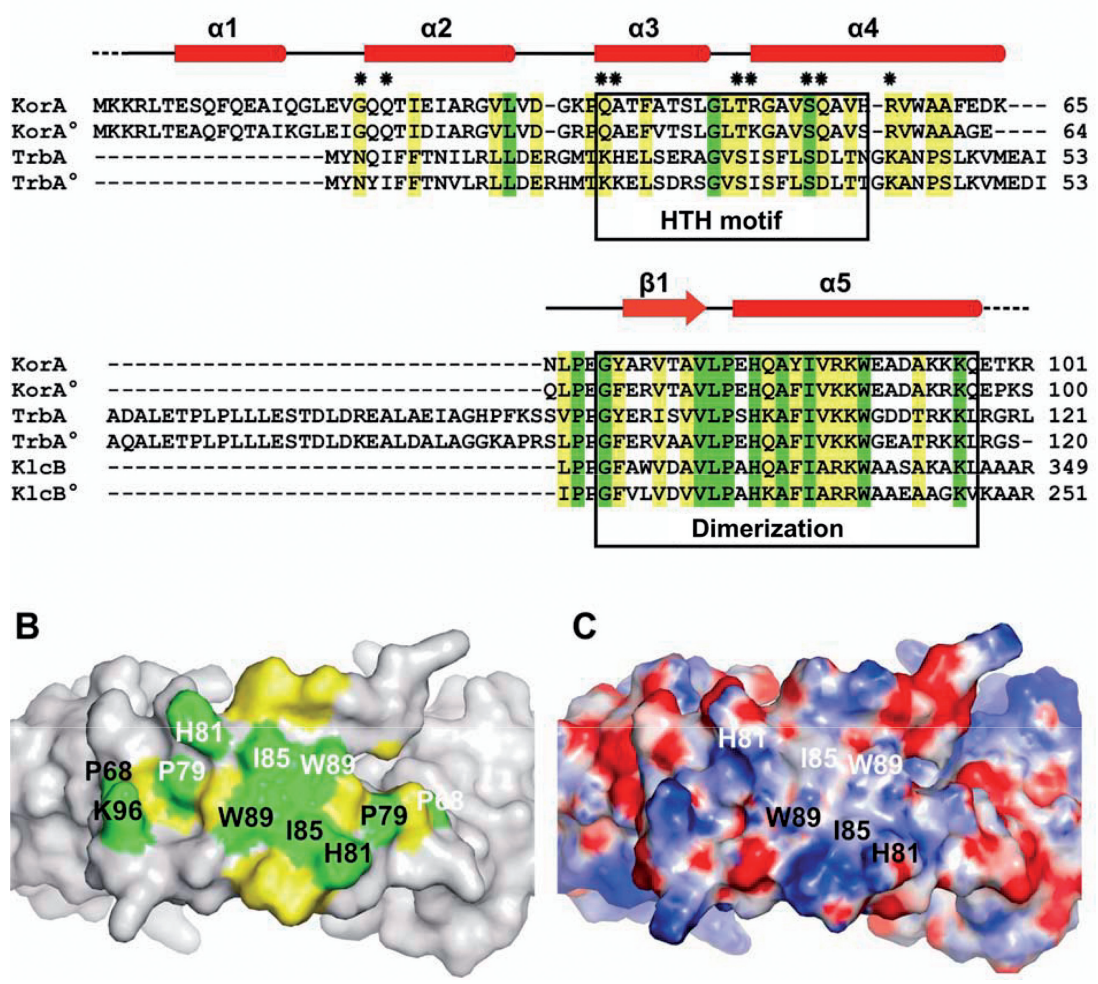

Figure 3. The dimerization module of KorA. (A) Multiple sequence alignment of RP4 and R751 (R751 members marked with ${ }^{\circ}$ ) proteins KorA, TrbA and KlcB (accession numbers as listed above and shared identity toward full-length KorA $\mathrm{RP}_{4}$ : P03052, 100\%; Q57423, 79\%; Q01716, 51\%; Q56468, 59\%; P52605, 32\%; and P52604, 41\%). The secondary structure elements of KorA are represented above the aligned sequences. Identical and similar residues are highlighted in green and yellow, respectively. Dots mark KorA residues specifically involved in DNA interaction. Sequences corresponding to the HTH or dimerization motifs are enclosed in boxes. (B) The molecular surface of the KorA dimerization region (top view) reveals a patch of highly conserved residues. Side chains of identical and similar residues, as seen in the sequence alignment (A), are highlighted in green and yellow, respectively. Residues of one KorA monomer are labeled with the same color (black or white). (C) Electrostatic surface potential of the KorA dimerization region (top view); positive charge in blue, negative charge in red. Residues possibly involved in protein-protein interactions are labeled in black and white. 
three-dimensional structures. The repressor proteins KorA and TrbA share a predicted HTH motif. Sequence conservation within this motif is limited, reflecting the different operator sequences on the RP4 or R751 genomes to which the repressors bind. A strikingly high degree of sequence conservation characterizes the dimerization module with identical residues at 10 sequence positions between Pro68 and Lys96 of KorA $\mathrm{RP}_{4}$. For the KorA and $\operatorname{TrbA}$ proteins this conservation is found at the C-terminus, $\mathrm{KlcB}_{\mathrm{RP} 4}$ and $\mathrm{KlcB}_{\mathrm{R} 751}$ include additional 116 or 154 residues, respectively, adjacent to this region.

Highlighting the preserved region on the exposed molecular surface of KorA reveals a large patch of identical and type-conserved amino acids at the top of the dimer region (Figure 3B). Positioned directly in the center are the exposed hydrophobic side chains of Ile85 and the polar aromatic residues Trp89, His81 and Tyr84 of each monomer (Figure 3C). All four residues are known to favor protein-protein interface locations (35). Together, they create a surface patch which could serve as the site of interaction with KorB, a finding, which is consistent with a recent NMR study that identified Tyr84 as a critical residue for KorA-KorB cooperativity (15). It would seem formally possible that, alternatively, protein interactions would involve the opposite, inner side of the dimerization region which faces the DNA. In KorA, the distance between this inner surface and the DNA helix is large enough to permit further intermolecular interactions, also possible for TrbA, which seems to include a longer linker region, resulting in a considerably increased diameter of its ring-like assembly. KlcB has no predicted DNA-binding ability and therefore retains no ring-like conformation. However, in the KorA structure the exposed residues of the inner side of the dimerization module are Arg73 and Thr75 (Figure 2B). These residues show no conservation in our alignment (Figure 3A). We conclude, therefore, that the outer surface of the dimerization regions of KorA, TrbA and $\mathrm{KlcB}$ serves as contact site for the flexible and structurally unknown N-terminal domain of KorB.

\section{DNA-binding domain}

In the DNA-binding domain of KorA four compactly folded helices $(\alpha 1-\alpha 4)$ are arranged around a core of hydrophobic residues, oriented toward the interior of the bundle. Sharp turns between the four helices are mediated by three glycine residues, Gly16, Gly34 and Gly45. Helices $\alpha 1$ and $\alpha 3$ sandwich $\alpha 2$, all three being positioned on one side of $\alpha 4$. The three $\mathrm{N}$-terminal helices are tethered to $\alpha 4$ by van der Waals interactions, stabilizing the relative orientation of the scaffold and recognition helices ( $\alpha 3$ and $\alpha 4)$ of the HTH motif. Glu12 of $\alpha 1$ forms hydrogen bonds with the C-terminal Lys65 and Asn66 side chains of $\alpha 4$, and also the Thr23 ( $\alpha 2)$ and Arg57 ( $\alpha 4)$ side chains are hydrogen bonded.

Structural similarity searches using DALI (36) found no homolog for the full-length KorA. If the search is limited to the DNA-binding unit (residues 2-65) a good resemblance to the YlxM/p13-like family can be found, such as the hypothetical UPF0122 transcription factor SAV1236 from Staphylococcus aureus (PDB entry 1xsv) with a DALI Z-score of 7.7 and an r.m.s. deviation of $2.2 \AA$. If the search is focused on the DNA recognition region, the orientation of the scaffold and the recognition helix reveal structural homology to the C-terminal $\sigma_{4}$ domain of FliA $\left(\sigma^{28}\right)$ from Aquifex aeolicus (1rp3) and to the C-terminal HTH motif of the GerE-like TraR from Agrobacterium tumefaciens $(1 \mathrm{~h} 0 \mathrm{~m})$ with $Z$-scores of 4.1 and 3.6 , and r.m.s. deviations of $1.3 \AA$ and $1.8 \AA$, respectively. Sigma factors and members of the GerE-like family are key regulators of bacterial transcription; $\sigma_{4}$ specifically recognizes the -35 promoter element with its HTH motif.

\section{Orientation of the operator site}

The synthetic 18-bp $\mathrm{O}_{\mathrm{A}}{ }^{*} \mathrm{DNA}$ duplex is perfectly symmetric within the 12-bp operator site. The $3 \mathrm{bp}$ on both ends outside the consensus sequence lack in symmetry. They contain three brominated deoxyuridine nucleosides at positions 2, 16 and 17 on the antisense strand (Figure 4A). Upon analyzing the heavy-atom substructure, six bromine sites were detected, permitting to fit the DNA into the electron density in two alternative orientations. After refining the complex with only one orientation present, prominent difference density at the terminal base pairs occurred (Figure 4C). The DNA thus adopts a 2-fold disorder with $\sim 50 \%$ occupancy for each orientation (Figure 4B). The structures of the two oligonucleotides (strands EF and $\mathrm{GH}$ ) superimpose well with an r.m.s. deviation of $1.068 \AA$ for all atoms, as calculated with LSQKAB (26). Within the symmetric 12-bp operator site the r.m.s. deviation reduces to $0.659 \AA$ for all atoms. In the crystal, the 18-bp oligonucleotides are stacked with negative twist at the junction along the crystallographic $b$ axis, giving rise to a pseudo-continuous double helix (Figure 1C).

\section{The KorA dimer binds to the operator site}

KorA binds the 18-mer DNA as a dimer, with each monomer contacting one half-site of the operator fragment. The electrostatic surface potential of the DNA-binding domain reveals a positively charged patch facing the bound DNA (Figure 4B). KorA recognizes the operator sequence specifically through the recognition helix, which is inserted into consecutive major grooves of the operator site, as observed in many prokaryotic HTH-containing proteins. The N-terminus of the recognition helix is imbedded in the major groove making direct base contacts through its side chains. The recognition helix is oriented perpendicular to the helical axis of the DNA and profoundly penetrates into the major groove. The DNA is bent so as to allow full access of KorA side chains into the adjacent major grooves. The position of the recognition helix is stabilized by hydrogen bonds between residues at the $\mathrm{N}$-termini of helices $\alpha 1-\alpha 3$ and the sugarphosphate backbone of the DNA, sealing the major groove above the contact site of the recognition helix. Contacts to the minor groove of the DNA are not observed. 

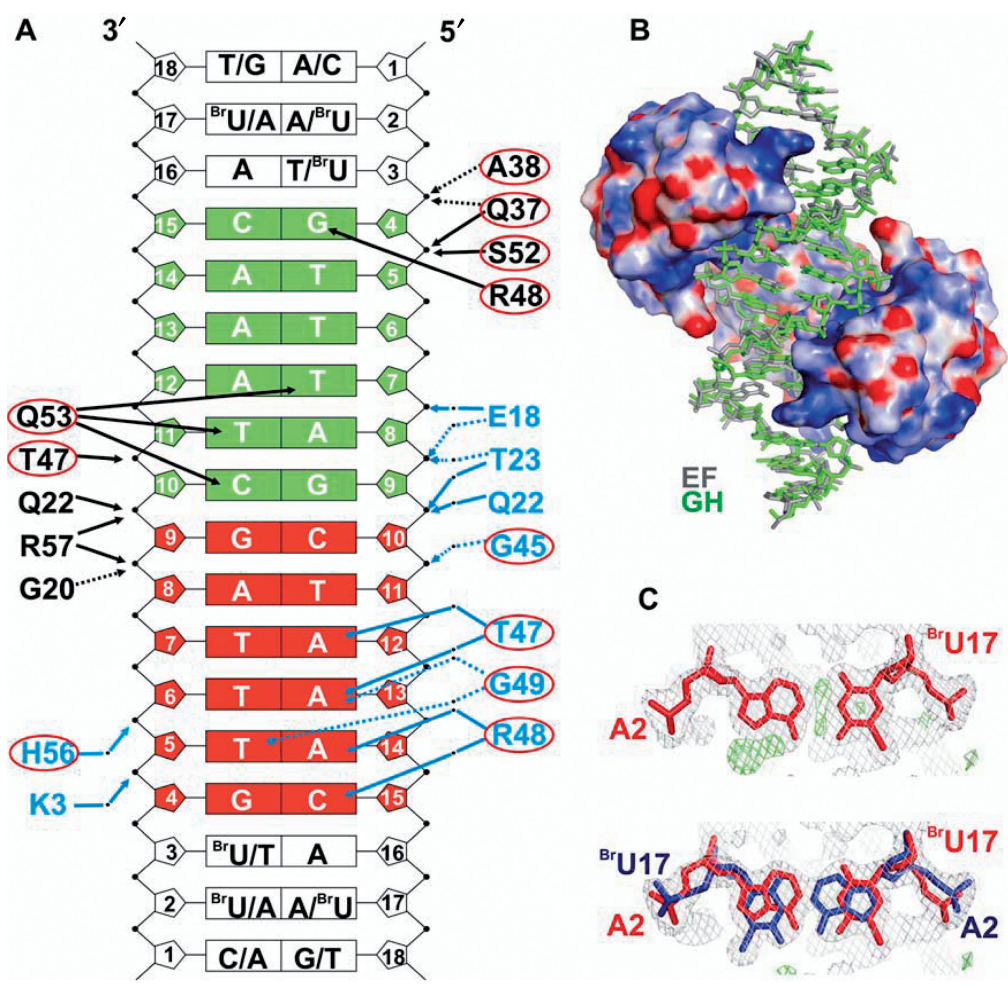

Figure 4. KorA binds its operator binding site. (A) $\mathrm{O}_{\mathrm{A}}{ }^{*}$ with the central 12-bp consensus operator sequence in red and green. (In $\mathrm{O}_{\mathrm{A}}$ the ${ }^{\mathrm{Br}} \mathrm{U}$ bases are replaced by T.) The symmetric operator has two half-sites, each of which binds one KorA monomer in identical geometry. Two different types of protein-DNA interactions are shown in the two half-sites. In the top half-site (green), only direct protein-DNA contacts are shown, whereas watermediated interactions are shown in the bottom half-site. Both types of interactions occur simultaneously in both half-sites of the operator DNA. Amino acids circled in red are from the HTH motif of KorA. Direct hydrogen-bonded contacts, involving protein side chains and DNA, are indicated by black solid arrows, those between protein backbone and DNA by black dotted arrows. Contacts mediated by water molecules are marked by blue arrows, those between protein side chains and DNA by blue solid lines, and those between protein backbone and DNA by blue dotted lines. (B) Electrostatic surface potential of KorA; positive charge of the protein in blue and negative charge in red. The DNA is bound to the KorA dimer in two orientations with duplexes EF (gray) and GH (green). (C) Two-fold disorder of KorA-bound $\mathrm{O}_{\mathrm{A}}{ }^{*} \mathrm{DNA}$. Left, base pair A2- ${ }^{\mathrm{Br}} \mathrm{U} 17$ with electron density before allowing for 2-fold disorder of the KorA-bound DNA. Right, A2- ${ }^{\mathrm{Br}} \mathrm{U} 17$ base pairs of the superimposed DNA duplexes EF (red) and GH (blue) after refinement. The $2 F_{\mathrm{o}}-F_{\mathrm{c}}$ density (gray) is contoured at $1.0 \sigma$, the $F_{\mathrm{o}}-F_{\mathrm{c}}$ difference density (green) is contoured at $3.0 \sigma$.

\section{Arg48 and GIn53 are essential for specific DNA binding}

In the structure each KorA monomer engages in a total of 27 identical contacts, of which 13 are direct and 14 are water mediated (Figure 4A). The 2-fold disorder of the DNA does not affect protein-DNA interactions, because KorA contacts only the perfectly palindromic 12-bp operator sequence and not the flanking three terminal base pairs, which lack in symmetry.

The specificity of KorA for its $\mathrm{O}_{\mathrm{A}}$ site is primarily based on two side chain interactions from the recognition helix $(\alpha 4)$ reaching deep into the major groove to make several base contacts (Figure 5A, Table 2). The Arg48 side chain binds to the $\mathrm{O}^{6}$ and $\mathrm{N}^{7}$ atoms of $\mathrm{G} 4$, and $\mathrm{G} \ln 53$ contacts the three bases T7 $\left(\mathrm{O}^{4}\right), \mathrm{C} 10\left(\mathrm{~N}^{4}\right)$ and $\mathrm{T} 11\left(\mathrm{O}^{4}\right)$. This is consistent with the finding that the seven KorA operators fall into two classes. Class I operators (korAp, klaAp and $\operatorname{trf} A p)$ are perfectly symmetric with $K_{\mathrm{D}}$ values of $13-20$ $\mathrm{nM}$ while class II operators ( $k l c A p, k f r A p, k l e A p$ and $k l e C p$ ), with more then 10 -fold higher $K_{\mathrm{D}}$ values, are lacking either G4 (contacted by Arg48) or T7 (contacted by Gln53) in one half-site of the consensus sequence (12). Each specific contact is made to the up- and to the down-oriented DNA duplex with very similar hydrogen bond lengths (Table 2).

The importance of specific contacts between KorA and the operator $\mathrm{O}_{\mathrm{A}}$ is supported by electrophoretic mobility shift assays. Four KorA variants were designed. In two mutants exactly one of the two specific DNA-binding residues was changed to alanine (R48A and $\mathrm{Q} 53 \mathrm{~A})$, in a third we replaced both specific binders (R48A/Q53A). According to the binding observed in the crystal structure (Figure 5A), truncation of the Arg48 side chain would remove two hydrogen bonds per operator half-site, and the Q53A mutation would lead to the loss of three hydrogen bonds per half-site. Furthermore, we changed Gln53 to the isosteric Glu53, causing the loss of two out of three hydrogen bonds.

We purified wt and mutants of KorA and tested for binding to a 436-bp DNA duplex containing the class I $\mathrm{O}_{\mathrm{A}}$ site of korAp (Figure 5B). wt KorA associates specifically with $\mathrm{O}_{A}$, and no retardation was observed with the nonspecific control DNA fragments that do not carry an $\mathrm{O}_{\mathrm{A}}$ site. The introduction of Q53A, R48A/Q53A and $\mathrm{Q} 53 \mathrm{E}$ mutations completely abolished the ability 
A

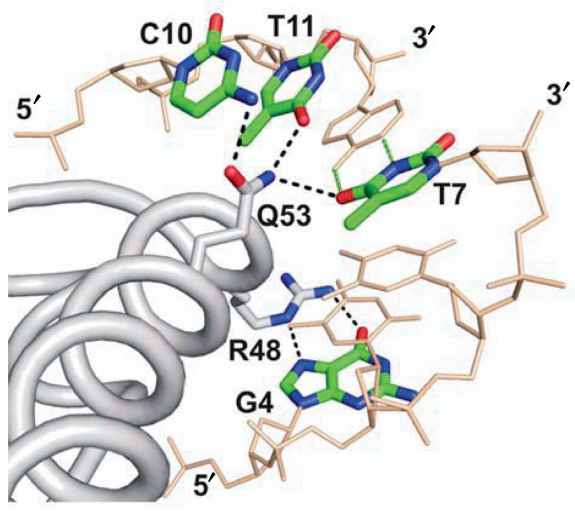

B

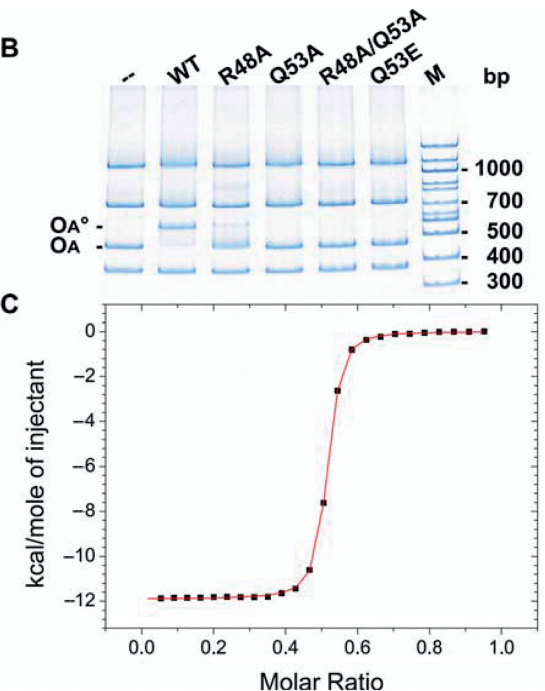

Figure 5. Specific binding of KorA to its operator-binding site. (A) Specific KorA-O ${ }_{A}^{*}$ contacts in the crystal. Arg48 and Gln53 of the recognition helix $(\alpha 4)$ of one KorA monomer form specific hydrogen bonds to G4, T7, C10 and T11, in one half-site of the symmetric operator sequence. (B) In vitro binding assay of purified wt KorA and mutants binding to DNA. The 436-bp fragment carries the class I $\mathrm{O}_{\mathrm{A}}$ site (kor $\left.A p\right)$ of plasmid RP4, the other fragments serve as competitor DNA; 7.5 pmol of wt and each mutant protein were applied. The DNA mixture contained 0.15 pmol of each fragment. $\mathrm{O}_{\mathrm{A}}$, DNA fragment containing $\mathrm{O}_{\mathrm{A}} ; \mathrm{O}_{\mathrm{A}}{ }^{\circ}$, complex of $\mathrm{O}_{\mathrm{A}}$ with wt KorA; M, 100-bp DNA ladder. (C) Calorimetric titration of KorA with $18-\mathrm{bp} \mathrm{O}_{\mathrm{A}}$. The panel shows the integrated heat released after correction of dilution (data points, squares) and the curve of best fit (red) for one KorA dimer binding to a single $\mathrm{O}_{\mathrm{A}}$ site.

Table 2. Specific binding of $\mathrm{Arg} 48$ and $\mathrm{Gln} 53$ to $\mathrm{O}_{\mathrm{A}}$

\begin{tabular}{lll}
\hline KorA & $\mathrm{O}_{\mathrm{A}}{ }^{*}$ & Bond lengths $(\AA)^{\mathrm{a}}$ \\
\hline $\mathrm{R} 48\left(\mathrm{~N}^{\eta 2}\right)$ & $\mathrm{G} 4\left(\mathrm{O}^{6}\right)$ & $3.11 \pm 0.15$ \\
$\mathrm{R} 48\left(\mathrm{~N}^{\varepsilon}\right)$ & $\mathrm{G} 4\left(\mathrm{~N}^{7}\right)$ & $3.08 \pm 0.11$ \\
$\mathrm{Q} 53\left(\mathrm{O}^{\varepsilon 1}\right)$ & $\mathrm{C} 10\left(\mathrm{~N}^{4}\right)$ & $3.07 \pm 0.31$ \\
$\mathrm{Q} 53\left(\mathrm{~N}^{\varepsilon 2}\right)$ & $\mathrm{T} 11\left(\mathrm{O}^{4}\right) \mathrm{T} 7\left(\mathrm{O}^{4}\right)$ & $2.92 \pm 0.062 .90 \pm 0.17$ \\
\hline
\end{tabular}

${ }^{\mathrm{a} C}$ Calculated from four different bond lengths derived from contacts of the symmetric KorA dimer to the 2-fold disordered 18-bp $\mathrm{O}_{\mathrm{A}}^{*}$ DNA.

of KorA to bind specifically to $\mathrm{O}_{\mathrm{A}}$ in all cases. R48A retains reduced capability to shift the operator fragment and shows some nonspecific binding in addition. As a whole, the gel-shift assays strongly suggest that the binding mode observed in the crystal reflects the mode of operator recognition by KorA in solution and identifies Gln53 as the crucial residue for specific operator binding.

To validate the strong binding capacity of wt KorA, we performed ITC-binding studies using the nonbrominated 18-mer duplex $\mathrm{O}_{\mathrm{A}}$ containing the class I operator sequence. ITC data yielded $K_{\mathrm{D}}$ values of $23.3 \pm 0.7 \mathrm{nM}$ for KorA:DNA (2:1) binding at $37^{\circ} \mathrm{C}$ (Figure 5C).

\section{DNA conformation of $\mathbf{O}_{\mathrm{A}}$}

CURVES 5.3 analysis (37) shows that the DNA in the crystal is smoothly bent by $14^{\circ}$ and adopts, essentially, a standard B-DNA form with a mean twist of $33.3^{\circ}$ and rise of $3.3 \AA$ per base-pair step. The major grooves are widened from 11 to $15 \AA$ upon insertion of the recognition helix. The binding of the Arg48 side chain to the G4 base is associated with a local overwinding of the DNA helix at the G4-T5/A14-C15 and the T5-T6/A13-A14 base-pair steps (twist values of $36^{\circ}$ and $42^{\circ}$, respectively). Similarly, the Gln53 binding to bases T7, C10 and T11 is linked with a slight overwinding of the helix at these base-pair steps (Figure 4A).

\section{DISCUSSION}

The expression of RP4 genes is tightly controlled, and reacts very sensitively to fluctuations in repressor levels. During exponential growth of the host there are $\sim 2000$ KorA and 1500 KorB dimers in the cell $(12,14)$ binding to three different classes of operator sites with different affinities. It is not clearly understood, if second- or third-class operators only play a supporting role in this control circuit, and how crucial cooperativity between repressor proteins really is. Interaction between repressor molecules probably increases association rates by helping proteins to locate and bind their operator sites more easily, especially at low protein concentrations. This is important, because KorA or KorB alone might arrest transcription of their own genes at the kor Ap site (class I) before any of the low-affinity binding sites are bound.

The structure of the KorA- $\mathrm{O}_{\mathrm{A}}{ }^{*}$ complex shows a remarkable structural complementarity between the repressor's DNA-binding domain and the 12-bp operator-binding site. KorA binds the palindromic DNA symmetrically with its HTH motif. Each monomer contacts the major groove on one half-site of $\mathrm{O}_{\mathrm{A}}$. The crystal structure indicates that the KorA dimer is very stable. This is supported by gel filtration and analytical 


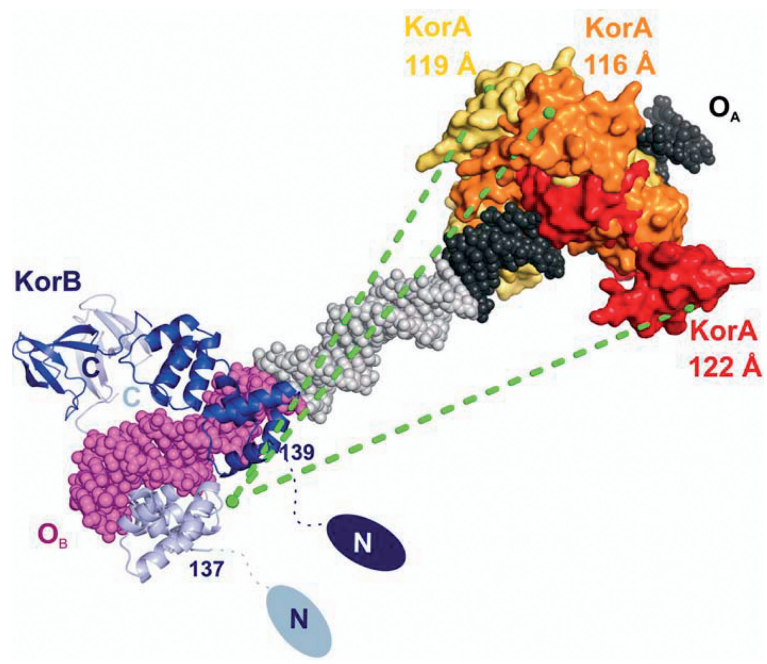

Figure 6. Model for the interaction between KorA and KorB at RP4 promoters. Crystal structures of the RP4 repressor proteins KorA (yellow, orange and red, each representing the molecular surface of one dimer, respectively) and KorB (chain A, blue, and chain B, light blue; PDB entries 1r71 for DNA-binding domain KorB-O, residues 137-252, and ligu for the C-terminal domain, residues 299-358) $(40,39)$ bound to their operator sites. $\mathrm{O}_{\mathrm{A}}$ (black) and $\mathrm{O}_{\mathrm{B}}$ (magenta) were joined by standard B-form DNA (gray), generated with MAKENA (http://structure.usc.edu/make-na/) representing the linker region at the korAp site. $\mathrm{O}_{\mathrm{A}}$ and $\mathrm{O}_{\mathrm{B}}$ midpoints are separated by $32 \mathrm{bp}$ $($ KorA $=$ yellow), by 33 bp ( $\operatorname{trfAp}$ and $k l a A p$, KorA $=$ orange) and by 36 bp $(k f r A p$, KorA $=$ red). The structurally unknown N-terminal residues $1-136$ of each KorB monomer are symbolized by two ellipsoids in blue and light blue. The distances between the KorB N-terminal region and the KorA dimerization domains in their different settings are indicated by green dashed lines.

ultracentrifugation experiments where KorA was always found to be dimeric (data not shown).

KorA $_{\mathrm{RP} 4}$ and KorA $\mathrm{R}_{51}$ (Figure $3 \mathrm{~A}$; sequence identity $79 \%$ ) recognize the exact same operator sequence but prefer binding to their own plasmid-specific $\mathrm{O}_{\mathrm{A}}$ sites which only differ in the flanking DNA sequences (38). All residues of KorA $\mathrm{R}_{\mathrm{RP}}$ seen to participate in direct protein-DNA contacts in the crystal structure (Figure 4B) are identical in both proteins except for Arg48, shown above to be important for operator recognition which is a lysine residue in $\mathrm{KorA}_{\mathrm{R} 751}$. Moreover, His56, involved in watermediated DNA interactions, is found to be a serine in $\mathrm{KorA}_{\mathrm{R} 751}$. Mutagenesis of $\mathrm{H} 56 \mathrm{~S}$ in $\mathrm{KorA}_{\mathrm{RP} 4}$ leads to an increased affinity for all operators, especially for $\mathrm{O}_{\mathrm{A} / \mathrm{R} 751}$ (38). Most likely, both KorA proteins exhibit the same fold. Replacing Arg48 by a long and flexible lysine residue would probably also result in specific recognition of the G4 base within the $\mathrm{O}_{\mathrm{A}}$ site, but one hydrogen bond will be lost. It seems unlikely, however, that Ser56 could contact the DNA phosphate backbone in the same way as His56. Therefore, the DNA needs to be bent more than seen in our crystal structure, and maybe the flanking sequences of the operator sites, AT-rich in RP4 and GC-rich in R751, are important for the extent of DNA bending. It remains noteworthy that both KorA repressors function only suboptimally on their own plasmids, but equally strong if compared to each other.
The structure of the KorA- $\mathrm{O}_{A}{ }^{*}$ complex provides the first view of a global transcriptional regulator of the RP4 plasmid that cooperates with the repressor protein KorB. Our findings reveal how KorA dimerizes and how the KorA dimer specifically contacts its operator site, suggesting a plausible model for the complicated repressor cooperation mechanism in RP4.

On the molecular surface of the KorA dimerization domain we identified a prominent patch of residues favoring protein-protein interactions, which are conserved in RP4 repressor proteins that interact with KorB. The crystal structure of the C-terminal dimerization region of KorB, KorB-C, with interlocking SH3-like domains (aa 299-358) (39), and the DNA-binding domain KorB-O (aa 137-252) (40) are known. The highly flexible N-terminal domain (aa 1-136) could not be crystallized. We propose that KorB contacts KorA via its mobile $\mathrm{N}$-terminus, which could permit repressor-repressor interactions over variable $\mathrm{O}_{\mathrm{B}}-\mathrm{O}_{\mathrm{A}}$ distances. The centerto-center distance between the operator sites varies from $32 \mathrm{bp}$ (korAp) to $33 \mathrm{bp}$ (trfAp and klaAp) and $36 \mathrm{bp}$ $(k f r A p)$. Assuming standard B-form, as seen in the KorA and KorB operator complexes, also for the linker DNA, the flexible N-terminal domains of KorB and the interacting surface on the KorA dimerization module are located at nearly opposite sides of the DNA helix in korAp (Figure 6). As the operator sites are moving further apart to 33 or $36 \mathrm{bp}$ in $\operatorname{trfAp} / k l a A p$ and $k f r A p$, the helix twist puts the interacting repressor moieties successively on the same side of the DNA, keeping the effective KorB-KorA separation approximately constant. Given the observed flexibility of the KorB N-terminus, this model may explain the cooperation between KorB and KorA at RP4 promoters with variably spaced $\mathrm{O}_{\mathrm{B}}$ and $\mathrm{O}_{\mathrm{A}}$ sites and may be a structural template for KorB's interaction with the $\mathrm{TrbA}$ repressors and $\mathrm{KlcB}$ that share the predicted interaction surface. The reported cooperation between KorA and KorB over a spacing of $92 \mathrm{bp}$ at the kleA promoter is not explained by this model and may require looping of the DNA as suggested by Bingle et al. (13).

\section{ACKNOWLEDGEMENTS}

We thank F. Skole and M. Terne for technical assistance, E.-C. Müller and A. Otto for mass spectrometry, J. Behlke (all Max-Delbrück Center, Berlin) for analytical ultracentrifugation, S. Keller and group (Research Institute for Molecular Pharmacology, Berlin) for useful discussions and help with the ITC experiment, and the staff at BESSY, Berlin, for beamline support.

\section{FUNDING}

The Fonds der Chemischen Industrie. Funding for open access charge: Max Delbrück Center for Molecular Medicine, Berlin-Buch.

Conflict of interest statement. None declared. 


\section{REFERENCES}

1. Adamczyk,M. and Jagura-Burdzy,G. (2003) Spread and survival of promiscuous IncP-1 plasmids. Acta Biochim. Polon., 50, 425-453.

2. Heinemann,J.A. and Sprague,G.F. Jr. (1989) Bacterial conjugative plasmids mobilize DNA transfer between bacteria and yeast. Nature, 340, 205-209.

3. Waters,V.L. (2001) Conjugation between bacterial and mammalian cells. Nature Genet., 29, 375-376.

4. Dennis,J.J. (2005) The evolution of IncP catabolic plasmids. Curr. Opin. Biotech., 16, 291-298.

5. Bahl,M.I., Hansen,L.H., Goesmann,A. and Sorensen,S.J. (2007) The multiple antibiotic resistance IncP-1 plasmid pKJK5 isolated from a soil environment is phylogenetically divergent from members of the previously established $\alpha, \beta$ and $\delta$ sub-groups. Plasmid, 58, 31-43.

6. Pansegrau,W. and Lanka,E. (1987) Conservation of a common 'backbone' in the genetic organization of the IncP plasmids RP4 and R751. Nucleic Acids Res., 15, 2385.

7. Pansegrau,W., Lanka,E., Barth,P.T., Figurski,D.H., Guiney,D.G., Haas,D., Helinski,D.R., Schwab,H., Stanisich,V.A. and Thomas,C.M. (1994) Complete nucleotide sequence of Birmingham Inc $\mathrm{P} \alpha$ plasmids. Compilation and comparative analysis. J. Mol. Biol., 239, 623-663.

8. Thorsted,P.B., Macartney,D.P., Akhtar,P., Haines,A.S., Ali,N., Davidson,P., Stafford,T., Pocklington,M.J., Pansegrau,W., Wilkins,B.M. et al. (1998) Complete sequence of the IncP $\beta$ plasmid R751: Implications for evolution and organisation of the IncP backbone. J. Mol. Biol., 282, 969-990.

9. Bingle,L.E. and Thomas,C.M. (2001) Regulatory circuits for plasmid survival. Curr. Opin. Microbiol., 4, 194-200.

10. Meyer,R. and Hinds,M. (1982) Multiple mechanisms for expression of incompatibility by broad-host-range plasmid RK2. J. Bacteriol., 152, 1078-1090.

11. Williams,D.R., Macartney,D.P. and Thomas,C.M. (1998) The partitioning activity of the RK2 central control region requires only inc $C$, kor $B$ and KorB-binding site $\mathrm{O}_{\mathrm{B}} 3$ but other KorB-binding sites form destabilizing complexes in the absence of $\mathrm{O}_{\mathrm{B}} 3$. Microbiology, 144, 3369-3378.

12. Jagura-Burdzy,G. and Thomas,C.M. (1995) Purification of KorA protein from broad host range plasmid RK2: Definition of a hierarchy of KorA operators. J. Mol. Biol., 253, 39-50.

13. Bingle,L.E., Macartney,D.P., Fantozzi,A., Manzoor,S.E. and Thomas,C.M. (2005) Flexibility in repression and cooperativity by KorB of broad host range IncP-1 plasmid RK2. J. Mol. Biol., 349, 302-316.

14. Chiu,C.M., Manzoor,S.E., Batt,S.M., Muntaha,S., Bingle,L.E. and Thomas,C.M. (2008) Distribution of the partitioning protein KorB on the genome of IncP-1 plasmid RK2. Plasmid, 59, 163-175.

15. Bingle,L.E., Rajasekar,K.V., Tul Muntaha,S., Nadella,V., Hyde,E.I. and Thomas,C.M. (2008) A single aromatic residue in transcriptional repressor protein KorA is critical for cooperativity with its coregulator KorB. Mol. Microbiol., 70, 1502-1514.

16. Kostelidou,K., Jones,A.C. and Thomas,C.M. (1999) Conserved C-terminal region of global repressor KorA of broad-host-range plasmid RK2 is required for co-operativity between KorA and a second RK2 global regulator, KorB. J. Mol. Biol., 289, 211-221.

17. Bhattacharyya,A. and Figurski,D.H. (2001) A small protein-protein interaction domain common to $\mathrm{KlcB}$ and global regulators KorA and TrbA of promiscuous IncP plasmids. J. Mol. Biol., 310, 51-67.

18. Balzer,D., Ziegelin,G., Pansegrau,W., Kruft,V. and Lanka,E. (1992) KorB protein of promiscuous plasmid RP4 recognizes inverted sequence repetitions in regions essential for conjugative plasmid transfer. Nucleic Acids Res., 20, 1851-1858.

19. Heinemann,U., Büssow,K., Mueller,U. and Umbach,P. (2003) Facilities and methods for the high-throughput crystal structural analysis of human proteins. Acc. Chem. Res., 36, 157-163.
20. Evans,G. and Pettifer,R.F. (2001) CHOOCH: a program for deriving anomalous-scattering factors from $\mathrm{X}$-ray fluorescence spectra. J. Appl. Crystallogr., 34, 82-86.

21. Kabsch,W. (1993) Automatic processing of rotation diffraction data from crystals of initially unknown symmetry and cell constants. J. Appl. Crystallogr., 26, 795-800.

22. Pape,T. and Schneider,T.R. (2004) HKL2MAP: a graphical user interface for phasing with SHELX programs. J. Appl. Crystallogr., 37, 843-844.

23. Cowtan,K. (1994) 'dm': an automated procedure for phase improvement by density modification. Jnt. CCP4/ESF-EACBM Newsl. Protein Crystallogr., 31, 34-38.

24. Lamzin,V.S., Perrakis,A. and Wilson,K.S. (2001) The ARP/wARP suite for automated construction and refinement of protein models. In Rossmann,M.G. and Arnold,E. (eds), International Tables for Crystallography. Volume F: Crystallography of Biological Macromolecules. Kluwer Academic Publishers, Dordrecht, The Netherlands, pp. 720-722.

25. Jones,T.A., Zou,J.Y., Cowan,S.W. and Kjeldgaard,M. (1991) Improved methods for building protein models in electron density maps and the location of errors in these models. Acta Crystallogr. A, 47, 110-119.

26. Kabsch,W. (1978) A solution for the best rotation to relate two sets of vectors. Acta Crystallogr., 32, 922-923.

27. Murshudov,G.N., Vagin,A.A. and Dodson,E.J. (1997) Refinement of macromolecular structures by the maximum-likelihood method. Acta Crystallogr. D, 53, 240-255.

28. Laskowski,R.A., MacArthur,M.W., Moss,D.S. and Thornton,J.M. (1993) PROCHECK: a program to check the stereochemical quality of protein structures. J. Appl. Crystallogr., 26, 283-291.

29. Hooft,R.W., Vriend,G., Sander,C. and Abola,E.E. (1996) Errors in protein structures. Nature, 381, 272.

30. DeLano,W.L. (2002) The PyMOL Molecular Graphics System, DeLano Scientific LLC, San Carlos, CA, USA.

31. Baker,N.A., Sept,D., Joseph,S., Holst,M.J. and McCammon,J.A. (2001) Electrostatics of nanosystems: application to microtubules and the ribosome. Proc. Natl Acad. Sci. USA, 98, 10037-10041.

32. Krissinel,E. and Henrick,K. (2007) Inference of macromolecular assemblies from crystalline state. J. Mol. Biol., 372, 774-797.

33. Kostelidou,K., Jagura-Burdzy,G. and Thomas,C.M. (1998) Mutational analysis of the global regulator KorA of broad-hostrange plasmid RK2. J. Mol. Biol., 281, 453-463.

34. Thompson,J.D., Higgins,D.G. and Gibson,T.J. (1994) CLUSTAL $\mathrm{W}$ : improving the sensitivity of progressive multiple sequence alignment through sequence weighting, position-specific gap penalties and weight matrix choice. Nucleic Acids Res., 22, 4673-4680.

35. Jones,S. and Thornton,J.M. (1997) Analysis of protein-protein interaction sites using surface patches. J. Mol. Biol., 272, 121-132.

36. Holm,L. and Sander,C. (1995) Dali: a network tool for protein structure comparison. Trends Biochem. Sci., 20, 478-480.

37. Lavery,R. and Sklenar,H. (1988) The definition of generalized helicoidal parameters and of axis curvature for irregular nucleic acids. J. Biomol. Struct. Dyn., 6, 63-91.

38. Kostelidou,K. and Thomas,C.M. (2002) DNA recognition by the KorA proteins of IncP-1 plasmids RK2 and R751. Biochem. Biophys. Acta, 1576, 110-118.

39. Delbrück,H., Ziegelin,G., Lanka,E. and Heinemann,U. (2002) An Src homology 3-like domain is responsible for dimerization of the repressor protein KorB encoded by the promiscuous IncP plasmid RP4. J. Biol. Chem., 277, 4191-4198.

40. Khare,D., Ziegelin,G., Lanka,E. and Heinemann,U. (2004) Sequence-specific DNA binding determined by contacts outside the helix-turn-helix motif of the ParB homolog KorB. Nature Struct. Mol. Biol., 11, 656-663.

41. Diederichs,K. and Karplus,P.A. (1997) Improved R-factors for diffraction data analysis in macromolecular crystallography. Nature Struct. Biol., 4, 269-275. 